\title{
Pharmacogenomics at the center of precision medicine: challenges and perspective in an era of Big Data
}

\author{
Dragan Primorac*, 1,2,3,4,5,6,7,8, Lidija Bach-Rojecky9 iD, Dalia Vađunec ${ }^{9}$, Alen Juginović ${ }^{2}$, \\ Katarina Žunić ${ }^{10}$, Vid Matišić11 ${ }^{11}$ Andrea Skelin ${ }^{1,12}$, Borna Arsov ${ }^{11}$, Luka Boban ${ }^{11}$, Damir \\ Erceg $^{1,7,8,13}$, Ivana Erceg Ivkošić1,8, Vilim Molnar ${ }^{11}$, Jasmina Ćatić1,5,14, Ivan Mikula ${ }^{1,15}$, \\ Ljubica Boban ${ }^{16}$, Lara Primorac ${ }^{17}$, Bernard Esquivel ${ }^{18}$ \& Morgan Donaldson ${ }^{18}$ \\ ${ }^{1}$ St Catherine Specialty Hospital, 10000 Zagreb \& 49210 Zabok, Croatia \\ ${ }^{2}$ University of Split School of Medicine, 21000 Split, Croatia \\ ${ }^{3}$ Eberly College of Science, 517 Thomas St, State College, Penn State University, PA 16803, USA \\ ${ }^{4}$ The Henry C Lee College of Criminal Justice \& Forensic Sciences, University of New Haven, West Haven, CT 06516, USA \\ ${ }^{5}$ University of Osijek School of Medicine, 31000 Osijek, Croatia \\ ${ }^{6}$ University of Rijeka School of Medicine, 51000 Rijeka, Croatia \\ ${ }^{7}$ Srebrnjak Children's Hospital, 10000 Zagreb, Croatia \\ ${ }^{8}$ University of Osijek Faculty of Dental Medicine \& Health, 31000 Osijek, Croatia \\ ${ }^{9}$ University of Zagreb Faculty of Pharmacy \& Biochemistry, 10000 Zagreb, Croatia \\ ${ }^{10}$ PLIVA Hrvatska, 10000 Zagreb, Croatia \\ ${ }^{11}$ University of Zagreb School of Medicine, 10000 Zagreb, Croatia \\ ${ }^{12}$ Genos Glycoscience Research Laboratory, 10000 Zagreb, Croatia \\ ${ }^{13}$ Croatian Catholic University, 10000 Zagreb, Croatia \\ ${ }^{14}$ Clinical Hospital Dubrava, Department of Cardiology, 10000 Zagreb, Croatia \\ ${ }^{15}$ University North, Nursing Department, 42000 Varaždin, Croatia \\ ${ }^{16}$ Children's Hospital Zagreb, 10000 Zagreb, Croatia \\ ${ }^{17}$ Wharton Business School, University of Pennsylvania, Philadelphia, PA 19104, USA \\ ${ }^{18}$ OneOme LLC, Minneapolis, MN 55413, USA \\ *Author for correspondence: office@draganprimorac.org
}

Pharmacogenomics (PGx) is one of the core elements of personalized medicine. PGx information reduces the likelihood of adverse drug reactions and optimizes therapeutic efficacy. St Catherine Specialty Hospital in Zagreb/Zabok, Croatia has implemented a personalized patient approach using the RightMed ${ }^{\circledR}$ Comprehensive PGx panel of 25 pharmacogenes plus Facor V Leiden, Factor II and MTHFR genes, which is interpreted by a special counseling team to offer the best quality of care. With the advent of significant technological advances comes another challenge: how can we harness the data to inform clinically actionable measures and how can we use it to develop better predictive risk models? We propose to apply the principles artificial intelligence to develop a medication optimization platform to prevent, manage and treat different diseases.

First draft submitted: 18 September 2019; Accepted for publication: 18 November 2019; Published online: 17 January 2020

Keywords: artificial intelligence $\bullet$ personalized medicine $\bullet$ pharmacogenomics $\bullet$ therapy optimization

\section{Pharmacogenomics as a cornerstone of personalized medicine}

According to England's National Health Service, personalized medicine can be defined as the tailoring of medical treatment to the individual characteristics of each patient. The approach relies on scientific breakthroughs in our understanding of how a person's unique molecular and genetic profile influences susceptibility to certain diseases and predicts which medical treatments will be safe and effective, and which ones will not [1].

The traditional path of drug development which has marked the 20th century, has been based on identifying therapies which target an entire population. However, over time, we have come to recognize that patients bear distinctive familial traits which cause variations in response to therapy, and we have tailored treatments based on these traits. Over the years, there has been a gradual paradigm shift from the traditional approach of 'one drug fits 
Table 1. Genes-drugs interaction with the highest level of clinical relevance (Clinical Pharmacogenetics Implementation Consortium guidelines recommend a change in prescribing of the affected drug based on evidence that includes consistent results from well-designed, well-conducted studies).

\begin{tabular}{|c|c|c|c|c|}
\hline Classification of genes & Example of genes & Drug examples & Effect & Ref. \\
\hline \multirow{5}{*}{$\begin{array}{l}\text { I. Genes involved in the first } \\
\text { phase of drug metabolism } \\
\text { - cytochrome P450 enzymes }\end{array}$} & CYP2C9 & Phenytoin, warfarin & \multirow{5}{*}{$\begin{array}{l}\text { Certain genotype may influence drug } \\
\text { substrate metabolism - consequent } \\
\text { changes in drug efficacy and safety }\end{array}$} & {$[18,19]$} \\
\hline & CYP2C19 & $\begin{array}{l}\text { Amytriptiline (es)citalopram, sertraline, } \\
\text { clopidogrel, voriconazole }\end{array}$ & & [20-25] \\
\hline & CYP2D6 & $\begin{array}{l}\text { Tricyclic antidepressants, atomoxetine, } \\
\text { codeine, tramadol, fluvoxamine, } \\
\text { paroxetine, ondansetron, tropisetron, } \\
\text { tamoxifen }\end{array}$ & & {$[20,24-27]$} \\
\hline & CYP3A5 & Tacrolimus & & [28] \\
\hline & CYP4F2 & Warfarin & & [19] \\
\hline $\begin{array}{l}\text { II. Genes involved in the second } \\
\text { phase of drug metabolism }\end{array}$ & UGT1A1 & Atazanavir & Safety - risk of hyperbilirubinemia & {$[29$} \\
\hline \multirow[t]{2}{*}{$\begin{array}{l}\text { III. Other genes involved in drug } \\
\text { metabolism }\end{array}$} & TPMT/NUDT15 & $\begin{array}{l}\text { Azathioprine }{ }^{\dagger} \text {, mercaptopurine }{ }^{\dagger}, \\
\text { thioguanine }^{\dagger}\end{array}$ & Efficacy and safety & [30] \\
\hline & $D P Y D$ & Capecitabine, tegafur, fluorouracil & Safety - increased drug toxicity & [31] \\
\hline IV. Genes for drug transporters & SLCO1B1 & Simvastatin & Safety - risk of myopathy & [32] \\
\hline \multirow[t]{2}{*}{ V. Genes for drug targets } & VKORC1 & Warfarin & Efficacy & [19] \\
\hline & CFTR & Ivacaftor ${ }^{\ddagger}$ & Efficacy & [33] \\
\hline \multirow{5}{*}{$\begin{array}{l}\text { VI. Genes for other proteins } \\
\text { important for drug action and } \\
\text { safety }\end{array}$} & $H L A-A$ & (Ox)carbamazepine & Safety - increased risk of SJS/TEN & [34] \\
\hline & $H L A-B$ & $\begin{array}{l}\text { (Ox)carbamazepine }{ }^{\ddagger}, \text { abacavir }^{\ddagger}, \\
\text { fosfo(phenytoin), allopurinol }\end{array}$ & Safety - increased risk of SJS/TEN & {$[18,35,36]$} \\
\hline & IFNL3 & Peginterferon-alfa $2 a$ and $2 b$, ribavirin & Efficacy & [37] \\
\hline & G6PD & Rasburicase $e^{\ddagger}$ & Toxicity - risk of hemolysis & [38] \\
\hline & CACNA1S/RyR1 & $\begin{array}{l}\text { Inhalational } \\
\text { anesthetics/suxamethonium }\end{array}$ & $\begin{array}{l}\text { Toxicity - increased risk of malignant } \\
\text { hyperthermia }\end{array}$ & [39] \\
\hline
\end{tabular}

all' to a more personalized, patient-oriented treatment $[2,3]$. Today, medicine embraces a more predictive, preventive, participatory and personalized approach to patients, health and diseases. This so-called P4 medicine faces a lot of challenges and barriers but offers just as many opportunities for the improvement of care and therapy outcomes [4].

Statistics demonstrate that over 4 billion prescriptions are issued each year in the US, however, only around $50 \%$ of them show the expected therapeutic efficacy. In the US alone, the direct and indirect cost of chronic pain management can range from $\$ 560$ to 635 billion annually [5-7]. Adverse drug reactions (ADRs) account for up to $7 \%$ of all hospital admissions, up to $20 \%$ of re-admissions and are the fourth leading cause of death. Their estimated cost is $\$ 136$ billion annually [8-11]. Genetic factors can account for up to $95 \%$ of an individual's drug response [12] and are estimated to contribute to as much as $20 \%$ of the total number of reported ADRs [8,9].

Historically, the concept of personalized medicine had its first mention in a monograph title in 1998 [13]. While some of the field's core concepts date back to the early 1960s, the basic idea can be credited to the Canadian physician, Sir William Osler (1849-1919) who recognized that no two individuals react or behave alike under the abnormal conditions we know as disease [14]. The advancement of new technologies, including SNP genotyping and microarray/biochips, have been among the key drivers for the implementation of personalized medicine in practice $[2,15,16]$.

Considering challenges in contemporary medicine, knowledge about the influence of genetic variation on drug response might be of great importance in predicting therapeutic outcome. Dosage adjustments according to the patient's genotype for certain drugs and drug classes have already been implemented into relevant clinical guidelines (Table 1). In fact, NIH-funded resource called the 'Pharmacogenomics Knowledgebase' (PharmGKB) is dedicated to the curation and dissemination of pharmacogenomic (PGx) information [17]. Drug labels containing PGx information are approved by regulatory agencies worldwide, including: the US FDA, the EMA and the Pharmaceuticals and Medical Devices Agency of Japan.

An accomplishment of PGx and personalized medicine that can be emphasized is therapy adjustment according to CYP450 polymorphisms [40]. This family of enzymes is responsible for the metabolism of the majority of drugs. 
Variations in highly polymorphic genes that code for some of the CYP enzymes influence their ability to metabolize certain drugs and consequently lead to differences in efficacy and safety in select patients. Poor metabolizers (PMs) have less active or inactive alleles that can cause drug overdose or an increase in toxicity. Conversely, ultrarapid metabolizers (UMs) who express multiple copies of a particular gene may experience lack of efficacy. The opposite effects are expected for prodrugs (e.g., clopidogrel, codeine), which can be non-effective in PM, while in UM, increased serum levels of active compounds along with the increased risk of toxicity are seen. Pharmaceutical companies investigate the metabolic pathways of potential new drugs in the early phases of drug development and it is now common for variations in CYP genes to be tested in patients during clinical trials [41,42].

The rise in 'omics' studies and exploration in recent years has generated more data whose interpretation requires methods well beyond the grasp of traditional statistical techniques. A massive influx of data relevant to disease symptoms, diagnostics, biomarkers, therapy, adverse effects - among others, has been collected over time. Thus, there is an obvious need for AI-powered platforms that allow the analysis and discovery of patterns in heterogeneous clinical data sets to improve overall patient care and ensure more prompt diagnostics.

Data-driven and networks-driven thinking and methods will play a critical role in the emergence of personalized healthcare [43]. Big Data is defined as "high-volume, high-velocity and high-variety information assets that demand cost-effective, innovative forms of information processing for enhanced insight and decision-making" [44]. The powerful merge between Big Data analytics and PGx will allow us to analyze, catalog, differentiate and utilize factors that contribute to a patient's drug response.

\section{Big Data in biomedicine: the emerging role of Al}

Artificial intelligence (AI) is making strides in various scientific fields, and biomedicine is no exception. The implementation of $\mathrm{AI}$ is of great interest in drug discovery, clinical trials, diagnostics, imaging and the overall healthcare system (i.e., risk management, remote patient monitoring, hospital management) [45]. Furthermore, the involvement of IT companies such as Google, Amazon or IBM into healthcare is becoming commonplace, applying AI principles to everything from medical devices to lifestyle management solutions [46].

Alan Turing conceptualized the idea of AI in the 1950s by developing a test which measured a machine's ability to exhibit intelligent behavior equivalent to that of a human [47]. During 1960s and 1970s computer programs ELIZA and PARRY were created by Joseph Weizenbaum and Kenneth Colby, respectively. While ELIZA, one of the first chatterbots, was developed to simulate conversations and give users an illusion of understanding, the PARRY implemented a crude model of the behavior of a paranoid schizophrenic [48]. In 1997 the AI concept gained more attention from the public, because the Deep Blue computer, specifically designed for playing chess, beat the famous chess player Kasparov [49]. Shortly thereafter, the core of AI became the training of computer algorithms to perform cognitive behavior (i.e., learning or problem solving) associated with human intelligence [45,46].

To understand AI, some of the basic concepts have to be identified. As an application of AI, machine learning (ML) aims to enable machines to perform their work skillfully by using intelligent software. Statistical learning methods constitute the backbone of intelligent software that is used to develop machine intelligence. The term machine learning means to enable machines to learn without programming them explicitly. There are four general subtypes of ML: supervised, unsupervised, semi-supervised and reinforcement learning methods. The objectives of ML are to enable machines to make predictions, perform clustering, extract association rules or make decisions from a given dataset [50].

While ML algorithms are based on predefined engineered features with explicit parameters based on expert knowledge, deep learning (DL) algorithms, as a subset of $\mathrm{ML}$, are designed to continually evaluate data with a logic structure analogous to how a human would make conclusions. To be able to do this, DL uses layers - algorithms called artificial neural networks (ANN), specifically deep neural networks (DNN). As such, ANN/DNN are more advanced to ML given their own rule-association and pattern discovery capacity. In contrast, if the ML algorithm makes a wrong outcome prediction, then a human has to act and make an adjustment to the core formula [45,51-53].

As part of the clinical decision algorithm, the presence and utilization of AI models in PGx implementation will be seen at different stages: from patient selection (stratification) to the changes in the prescription guidelines.

One of the critical components of the broad-scale implementation, and therefore, the democratization of PGx, will be using AI methods for the stratification of patients. This requires high-complexity data analytics for the categorization of patients into subpopulations which can be selected in the design of clinical trials or the clinical scenario. Such data includes: sociometric data, demographic data and heterogeneous biomedical data [54]. 


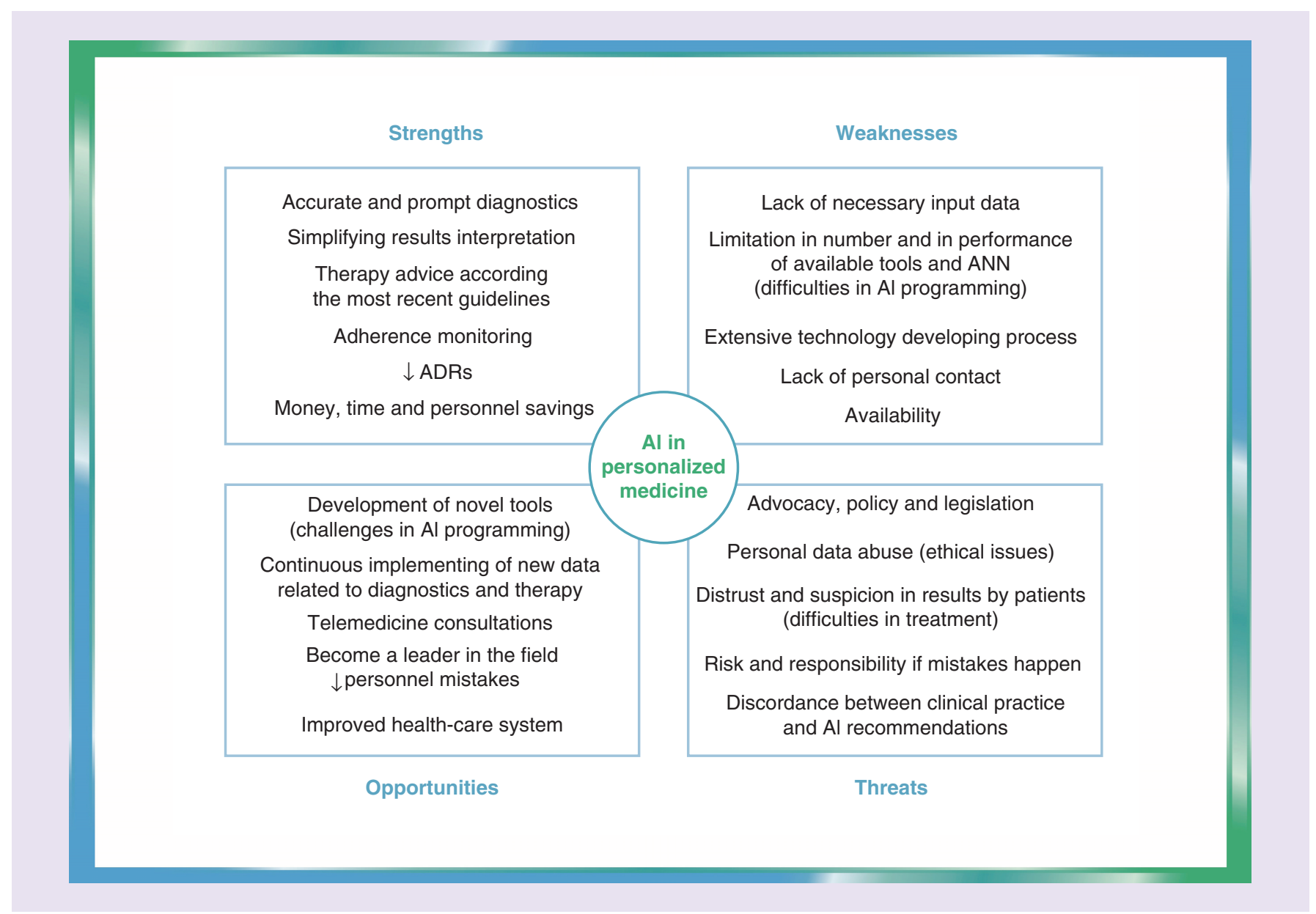

Figure 1. SWOT analysis of artificial intelligence implementation in clinical practice (in the context of pharmacogenomics). In this schematic view, the SWOT analysis summarizes all the features of introducing Al into clinical practice and patient care. ADR: Adverse drug reaction; ANN: Artificial neural network; Al: Artificial intelligence.

We believe that DL algorithms will play a significant role in overcoming the challenges associated with the implementation of PGx data in the electronic health record (EHR), as it will address the high dimensionality of the EHR data structure, noise, heterogeneity, sparseness, incomplete data fields, random error, systematic biases and the extraction of relevant clinical phenotypes among others. Related to PGx, DL could play a critical role in individual drug response prediction as well as in medication selection and dosing, all based on data analysed from big data databases [54].

In the following paragraphs we briefly analyze some strengths, weaknesses, opportunities and threats of AI implementation in clinical practice, especially in the context of PGx (Figure 1).

\section{Strengths}

In 2018, the FDA approved 14 algorithms for diagnostic image interpretation [45]. Additionally, in the Labowitz et al. study, the application of a sophisticated mobile application that used AI to visualize the patient and anticoagulant drug ingestion, resulted in adherence improvements in patients on anticoagulation therapy [55]. In biomedicine, especially in the patient care field where PGx is an integral component, AI can provide benefits on many levels (Figure 1.). For example: in therapy management (i.e., simplifying result interpretations, adherence monitoring) and risk predictions for pharmacologists or pharmacists; for healthcare systems in providing cost and time savings; and lastly, for patients so they can monitor their health status [45,46]. Cost-savings as a result of AI-related initiatives in healthcare are estimated to be $\$ 300$ billion per year in the US, or $0.7 \%$ of GDP [56]. 
Unsupervised ML algorithms can be deployed to discover patterns, correlations and structure that emerge from this Big Data. The use of AI in this dimension of the field of biomedicine is commonly seen and successfully applied in pharmacovigilance studies [57,58]. In addition, the combination of big data and ML algorithms could help with patient recruitment and selection for clinical trials but it could also enable patients to seek enrollment themselves [59].

\section{Weaknesses}

On the other hand, the limitations of AI have already been observed, and there are obstacles that need to be overcome (Figure 1). AI has to be powered by large data sets and the collection of such data can be challenging. Another challenge is to develop algorithms that can query heterogeneous data sets containing genomic, transcriptomic, proteomic and activomic data [60].

Though AI provides value to the clinical decision-making process, the importance of human intelligence, cannot be overstated. For example, although drug-drug interaction databases do exist as a clinical decision support tool, human interpretation is required to assess the many clinical factors that may impact a patient's drug response and to weigh the risks and benefits of certain drug combinations [60,61].

\section{Opportunities}

PGx represents one field of medicine where the application of ML algorithms can not only optimize medication choice and dosage but even predict ADRs. A most recent example demonstrated that validated PGx markers combined with a patient's clinical measures, specifically total baseline depression scores, could accurately predict SSRI remission/response in patients with major depressive disorder (MDD) [62]. Companies like OneOme (https: //oneome.com/home-international) are also applying ML principles to the development of a PGx risk stratification platform for patient populations. In this case, patients are assigned a PGx risk score based on medication history and utilization, level of evidence supporting drug-gene interactions for current medications as well as their clinical history of ADRs.

Additionally, AI could be used to process a large amount of data present in multifactorial diseases (i.e., psychiatric disorders). By processing available information, disease models could be built with predictable outcomes. By simultaneously analyzing data from various sources and at different points in time, a continuous model could be developed using updated content [63].

In surgical settings, AI algorithms could assist surgeons in pre- and post-operative care, decision-making as well as prevention of illnesses, providing an interdisciplinary approach to patient care. This includes interpretation of radiologic findings, pathologic findings, healing prognosis - among others [64].

In countries and populations with limited resources, AI could be a critical tool for improving healthcare. By assisting the predictive, diagnostic and therapeutic processes, AI becomes a powerful ally in a setting which is resource-limited. Furthermore, the digitalization of healthcare could be augmented by connecting the neural networks among countries with varying levels of development with the goal being the improvement of the general health of both the developing as well as developed countries [65].

\section{Threats}

There are several recognized issues and threats related to AI (Figure 1). For example, there is the infamous case of IBM's Watson making a dangerous recommendation in a particular oncology case. The patient was newly diagnosed with lung cancer and showed evidence of severe bleeding [66]. Watson's recommendation was a combination chemotherapy and bevacizumab, despite an FDA black box warning that the drug was contraindicated in patients experiencing severe bleeding. However, it must be taken into consideration that the algorithm was based on a small number of synthetic cases with very limited real data obtained from oncologists. This may raise a question about liability: is the developer or the operator responsible for making the wrong therapy recommendation? It was additionally argued that the state of AI hype has far exceeded the state of AI science, especially when it pertains to validation and readiness for implementation in patient care [45]. If AI were to be introduced into clinical practice prior to its optimal readiness, the potential mistakes might discourage the use of AI in its entirety.

Another important issue is related to potential breaches of protected health information and the possibility that this information might be used to cause harm. There has to be governmental legislation and proper policy and advocacy to mitigate such risks [45,46]. The General Data Protection Regulation (GDPR) has been implemented in the EU as a very strict policy that protects EU citizens from any harm resulting from data breaches. However, it 
is also an obstacle in some cases, preventing the full implementation of AI into the healthcare system [46]. There is also the issue of distrust and suspicion stemming from patients. How much time or how many generations have to be raised in a machine-trusted era to end the suspicion? [67].

It is also important to reassure that no algorithm is meant to replace a physician's expertise and experience; instead it must be seen as a supplementary clinical tool [68].

\section{The role of PGx in therapy optimization: opportunities \& limitations}

Although one might argue that the conventionally used 'trial-error' approach leads to positive clinical outcomes for the majority of treated patients, some of them may suffer from either a lack of therapeutic response or serious adverse reactions as demonstrated in the following examples.

Warfarin is a common anticoagulant used in clinical practice with highly variable response rates. Traditional clinical practice suggests that treatment be initiated at a fixed dose at first and adjusted according to the patient's international normalized ratio (INR). This takes time and requires full cooperation from the patient. Using PGx, warfarin dosing is made simpler and more reliable, as inter-individual variability in more than $60 \%$ of the patients can be explained by the analysis of CYP2C9, VKORC1 and CYP4F2 variants combined with the patient's age and weight $[3,69]$.

With a big data-driven tool, it is possible to integrate and even generate predictive impact models for most of the known factors that influence anticoagulation therapy response in patients, such as: age, race, height, weight, lifestyle, liver disease, amiodarone use, statin use, any ' azole' use, any 'sulfa' antibiotic, clinical indications, current INR, target INR, as well as the phenotype implications of CYP2C9, VKORC1, CYP4F2, all of which have an essential role in estimating the loading and maintenance dose of warfarin. DL algorithms have shown some advantages in this dosing process as well, including: high power and accuracy, ability to model nonlinear effects, interpretation of large genomic data sets, robustness to parameter assumptions and dispense with normal distribution test. For example, random forest regression, boosted regression tree and support vector regression models were utilized to predict warfarin maintenance dose in African-Americans. More than $70 \%$ of patients in low and median dose subgroups have been appropriately predicted with an ANN algorithm [70].

Another example in which the importance of PGx cannot be overstated, is in the case of severe cutaneous adverse reactions (SCARs). It was previously thought that SCARs are idiosyncratic or unpredictable. However, the relationship between $H L A$ alleles and drug-induced SCARs has been demonstrated for several drugs. One example is that of carbamazepine or phenytoin-induced Stevens-Johnson syndrome (SJS)/toxic epidermal necrolysis (TEN) associated with the $H L A-B^{*}$ 15:02 allele among the Han Chinese in Taiwan. Another one is allopurinol-induced SJS/TEN in patients with the $H L A-B^{*} 58: 01$ allele. With this drug-gene characterization, the prevention of SCARs is possible [71].

A recent case report in a pancreatic adenocarcinoma patient showed the importance of a personalized medicine approach. The patient was homozygous for the $U G T 1 A 1 * 28$ allele and a heterozygous carrier of a no-activity allele DPYD variant. Deficiencies in the enzymatic function of UGT1A1 and DPYD caused by genetic polymorphisms may result in decreased metabolism of irinotecan and 5-fluorouracil (5-FU), leading to increased risk for chemotherapy-induced toxicity. The patient had received a reduced dosage of FOLFIRINOX (chemotherapeutic protocol which consists of folic acid, 5-FU, irinotecan and oxaliplatin) and the dosage was adjusted based on the results of PGx testing. The treatment had been well-tolerated with no evidence of severe ADRs after the administration of five cycles. Importantly, at one of the last follow-ups, MRI examination showed a reduction in the size of the tumor. Without appropriate testing, the tolerability of the therapy and consequently, the efficacy, would have been compromised [72].

Moreover, genotype results might prove to be of crucial importance in better characterizing interethnic differences in drug response and may help to better inform drug development [73]. The availability of genetic information from diverse ethnic groups would serve to broaden the knowledge on population genetics and already documented differences in global and local populations, in addition to creating data for previously undocumented populations [74]. The discovery of population-distributed rare single nucleotide variations could also be achievable with a next-generation sequencing type of assay in the future [75].

Besides genome composition, other factors, such as epigenetic components and environmental inputs can affect the phenotype which leads to a change in the clinical drug response. Epigenetics is the study of heritable phenotype changes, through chromatin modifications and remodeling. The potential of epigenetic application in medicine 
is vast. It will be possible to test the relevance of epigenetic components in specific diseases, to exploit them as prognostic and diagnostic markers, and to use them as actionable targets for therapy [76].

Another example is the phenomenon of phenoconversion. For example, the co-administration of a drug that is a strong inhibitor of drug metabolism enzymes (DME) can significantly change the biotransformation rate of drug substrates of the respective enzymes. Thus, a person with the genotype of an extensive metabolizer (EM) could become a PM over time. Although this type of phenoconversion is transient, it can persist for weeks after strong inhibitors are discontinued. This is related to the half-life of the inhibitor and its affinity for the DME that it inhibits. Similarly, phenoconversion is also related to some diseases, like advanced cancer, where the future activity of CYP2C19 was found to be severely compromised, resulting in a PM status in $37 \%$ of the patients who had a normal genotype [77].

We should not forget the societal impact and broader perspective. Pharmacoeconomics data show that ADRs may cost the US healthcare system up to 30.1 billion dollars annually due to increased hospitalizations, extended hospital stays, additional clinical investigations and increased pharmacotherapy costs [78]. For these reasons alone, personalized prescribing should be considered as a cost-mitigation strategy. At the center of this personalized approach is PGx testing, which performed once can provide lifetime benefits. In fact, a 2016 systematic review of studies ( 80 studies in total) published between August 2010 and September 2014 investigating the cost-effectiveness of PGx screening tests showed that $55 \%$ of studies concluded that PGx testing was cost-effective, $16 \%$ of studies showed that PGx was cost-saving and 13\% showed that PGx was cost-dominant, which is defined as resulting in clinical benefits as well as cost-savings. Despite the study's findings that PGx testing is a cost-effective or costsaving strategy in most cases, we acknowledge the limitations and uncertainties in the studies themselves, including assumptions made with regards to the availability of tests results for clinical decision-making purposes, the cost of the PGx test and most importantly, the lack of clinical evidence supporting PGx testing [79].

An example of the potential of PGx in cost-saving can be found in its application in the choice of treatment of MDD. Due to the current trial-and-error treatment strategies, MDD presents an important opportunity to demonstrate the cost-effectiveness of PGx testing. MDD affects 16 million adults aged 15-44 in the US and is currently the leading cause of disability worldwide [80,81]. At current prevalence rates of $12-20 \%$ among all depressed patients in the US, treatment-resistant depression may present an annual added societal cost of $\$ 29-\$ 48$ billion, driving the total societal costs of MDD by as much as $\$ 106-\$ 118$ billion [82]. Due to the compounding effect of comorbid conditions, MDD is predicted to have the largest associated disease burden by the year 2030. Assuming a PGx test costs $\$ 2000$, annual cost-saving predictions are almost $\$ 4000$ per patient with PGx-guided medication management [80].

With many cost-effectiveness studies based on real-world data (e.g., based on claims databases or EHRs) still underway, mathematical modeling studies have made the case for multi-gene PGx panel testing. A recent Markov model developed by Technomics Research and OneOme, used a systemic review of the literature to estimate direct costs and estimated probabilities related to treatment-resistant major depression over the course of 1 year of a patient's life. Variables included: probability of adverse reaction to therapy, probability of prescription effectiveness, healthcare utilization probability (hospitalization, ED admission, etc.), healthcare utilization reimbursement (for ADR-related hospital stay, suicide-related hospital stay, follow-up visits, etc.) and antidepressant reimbursement. The average direct cost savings were estimated at $\$ 560$ and as high as $\$ 2093$ compared with standard of care. With indirect costs and comorbid benefits being out of scope for this study, the findings suggest that a moderately priced PGx test can generate substantial cost-savings in as little as a 1 year period [83].

The cost-effectiveness of PGx testing has been studied in other clinical areas as well. A decision-analysis model in patients with metastatic colorectal cancer showed that UGT1A1 genotype-guided dosing of irinotecan would result in $25 \%$ dose reductions in $11 \%$ of patients due to the UGT1A1*28 genotype, translating to potential savings of $\$ 7.96 \mathrm{M}$ across a population of 29,000 cases [84]. Not only has PGx-guided therapy been identified as cost-effective in treating patients with drugs like irinotecan, azathioprine, warfarin, citalopram, it can even be a cost-saving strategy [85].

In some fields of medicine, like in anesthesia, the implementation of PGx is still pending the development of additional evidence before broader implementation into clinical practice [86].

\section{Overview of PGx testing in St Catherine Specialty Hospital}

The concept of PGx-guided therapy integrates several levels which are interrelated, starting from DNA profiling and data analysis, using current molecular methods and bioinformatic tools, leading to result interpretation and 
Table 2. RightMed Comprehensive pharmacogenomics panel provides information on next 25 genes known to affect how patients respond to medications.

\begin{tabular}{|l|l|}
\hline $\begin{array}{l}\text { Involvement of genes in drug action } \\
\text { metabolism - cytochrome P450 enzymes }\end{array}$ & Genes of interest \\
\hline $\begin{array}{l}\text { Genes responsible for synthesis of enzymes involved in the second phase of drug } \\
\text { metabolism }\end{array}$ & $\begin{array}{l}\text { CPY1A2, CYP2B6, CYP2C9, CYP2C19, CYP2C Cluster, CYP2D6, CYP3A4, CYP3A5, } \\
\text { CYP4F2 }\end{array}$ \\
\hline $\begin{array}{l}\text { Genes responsible for synthesis of other enzymes that are important for drug } \\
\text { metabolism }\end{array}$ & DPYD, VKORC1, NUDT15 \\
\hline \begin{tabular}{l} 
Genes responsible for synthesis of drug transporters \\
\hline Genes responsible for synthesis of drug receptors
\end{tabular} & SLC6A4, SLCO1B1 \\
\hline Genes responsible for synthesis of other proteins important for drug function & HTR2A, HTR2C, DRD2, OPRM1, GRIK4, COMT \\
\hline
\end{tabular}

decision-making to offer the best available therapy. Continuous education of personnel involved in every step of this process is a prerequisite for the success of the personalized medicine concept.

St Catherine Specialty Hospital in Zagreb/Zabok, Croatia, which is pioneering the application of personalized medicine in clinical practice and where hundreds of patients have received PGx testing, understands the importance of moving into big data-driven clinical support algorithms as the next step to enhancing their internal clinical process. Essential to this process is ensuring interactive feedback between clinical end-users (physicians and other healthcare providers) and developers, so that stratification algorithms can be adapted to new insights in disease biology, new molecular data and changes in clinical settings [87].

At St Catherine Specialty Hospital, patients who are referred for PGx testing are first interviewed by a clinical specialist during a routine checkup visit. Patients receive detailed information about the testing protocol and are asked to sign an informed consent form. During the interview a detailed information about personal and family medical history, specific reason for PGx testing, body mass index, history of allergies, ADR details, a complete list of drugs including dosage and method of application, over the counter (OTC) drugs, food supplements and even specific food, as well as tobacco and alcohol consumption is collected. Following that, a buccal sample is taken in order to obtain DNA from the patient. OneOme's RightMed test kit (ORACollectDx, DNA Genotek) is composed of a $1 \mathrm{ml}$ saliva collection tube. Each tube has a unique barcode which is assigned to a specific patient and recorded in the test requisition form along with the patient's demographic data. Collected patient data is centralized in OneOme's database portal (https://portal.oneome.com/orders/). Once collected, the sample is sent in accordance with the provided kit instructions to OneOme's in-house CLIA/CAP-accredited laboratory. The DNA is then analyzed using the RightMed ${ }^{\circledR}$ panel (Table 2), developed by OneOme in collaboration with Mayo Clinic and brought to clinical practice by St Catherine Specialty Hospital. This approach to PGx testing determines SNPs using a TaqMan real-time PCR method and CNV analysis. Results of the DNA analysis are generated in individual test reports available in OneOme's HIPAA-secure portal. OneOme's data curation and clinical annotations are performed by a team of clinical pharmacists and scientists, supported by scientific evidence that meets OneOme's rigorous inclusion criteria and profesional guidelines (e.g., Clinical Pharmacogenetics Implementation Consortium (CPIC), Dutch Pharmacogenomics Working Group, French National Pharmacogenetics Network /Group of Clinical Onco-Pharmacology and Canadian Pharmacogenomics Network for Drug Safety).

Results of PGx testing are then interpreted by a genetic counseling team. The clinical decision support tool used for drug-gene interaction interpretation as well as possible drug-drug/food/supplements interaction is RightMed ${ }^{\circledR}$ Advisor [88]. The interpreted results and clinical recommendations are then presented to the patient by his or her attending clinician, whose role is to advise on the best pharmacological option based on the main principles of individualized medicine: the right drug for the right patient at the right time (Figure 2).

\section{Clinical cases from St Catherine Specialty Hospital Clinical case I}

A 66-year-old patient with benign prostate hypertrophy, arterial hypertension, diabetes mellitus type II who suffered from acute coronary syndrome with stent replacement in his right coronary artery (RCA) has come to the hospital complaining of nausea and unsatisfactory blood pressure regulation with lisinopril. Bisoprolol, metformin, rosuvastatin, clopidogrel were the other drugs included in his therapeutic regimen. The patient's PGx status was assessed. He had CYP2C19 ${ }^{*} 1 /{ }^{*} 2$ genotype which corresponds to a phenotype of an intermediate metabolizer. 
(A)

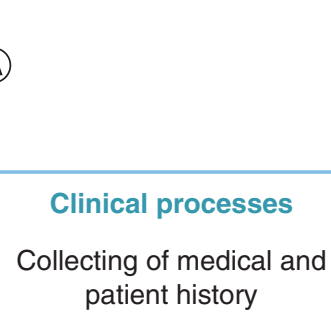

Buccal (cheek) swab sampling for RightMed ${ }^{\circledR}$ test

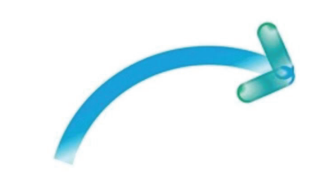

(B) Genetic analysis and
data processing

RightMed $^{\circledR}$ panel comprehensive test

RightMed $^{\circledR}$ advisor

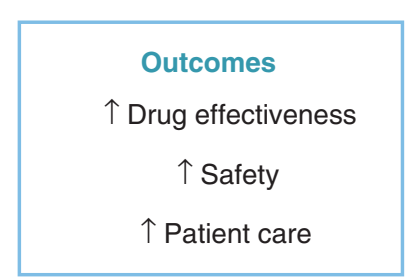

(D)

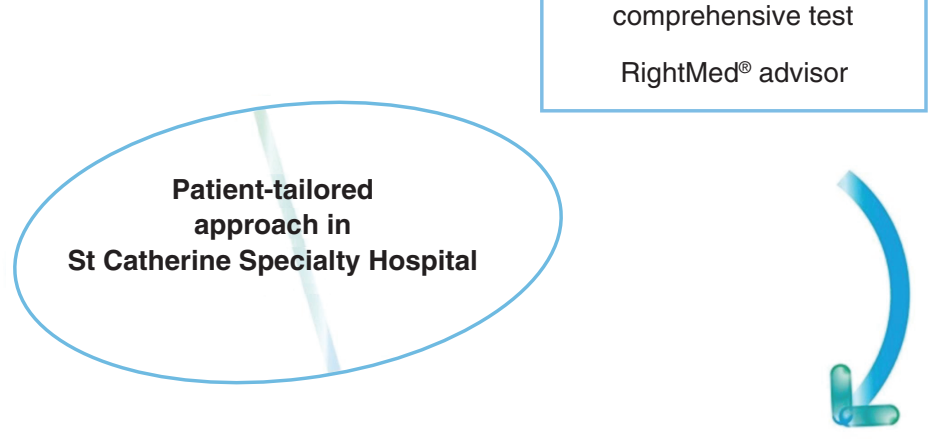

Clinical interpretation of results and recommendations Multi disciplinary team approach RightMed ${ }^{\circledR}$ advisor

(C)

Figure 2. A schematic representation of implementation personalized pharmacogenomic based treatment and healthcare system. (A) The first step is to collect medical and patient history via a specific form followed by a buccal swab for DNA sampling for RT-PCR. (B) RightMed ${ }^{\circledR}$ panel comprehensive test processes the pharmacogenomic results using an algorithm. RightMed ${ }^{\circledR}$ Advisor is being generated here. (C) Results are then interpreted by a multi-disciplinary team. In addition to drug-gene interactions, the RightMed ${ }^{\circledR}$ Advisor platform checks for drug-drug, drug-food (supplement) interactions. (D) The results aid in providing better patient care, superior therapy outcome and greater drug effectiveness while at the same time reducing the rate of adverse drug reactions.

The implication of this variant is reduced conversion of clopidogrel to its active metabolites and therefore reduced platelet inhibition and a higher risk for recurrence of a cardiovascular event. Here, proper platelet inhibition is of great importance due to one stent already replaced in RCA.

It is also important to mention that pantoprazole inhibits CYP2C19 and additionally decreases the levels of clopidogrel's active metabolites. The patient was advised to initiate therapy with prasugrel or ticagrelor, instead of clopidogrel, as they do not undergo the same metabolism and do not have the reported drug-drug interactions with pantoprazole [89]. Furthermore, clopidogrel increases levels of rosuvastatin which could potentially lead to myopathy, acute renal injury and liver damage [90]. Lisinopril, an angiotensin converting enzyme inhibitor included in his daily therapy can increase risk of hypoglycemia [91]. Therefore, the patient was advised to monitor blood sugar levels closely if he continues to use the above-mentioned drugs concurrently.

\section{Clinical case II}

A 75-year-old patient had a fracture of the distal radial bone with the development of osteoporosis on the same arm which led to painful sensations. Ibuprofen was introduced as a drug of choice for pain management, however patient's pain was not managed adequately. Therefore, a combination of tramadol and dexketoprofen was recommended. Pantoprazole, metformin and rosuvastatin were also included in her therapeutic regimen. After 
coming to the hospital, her PGx profile was assessed. The patient's CYP2D6 $* 4 /{ }^{*} 4 * 68$ genotype corresponded to a PM phenotype, while the other genotype CYP2C19 ${ }^{*} 2 /{ }^{*} 17$ corresponded to an intermediate to normal metabolizer phenotype. Due to the poor activity of CYP2D6, we predicted that tramadol would produce little therapeutic response since its biotransformation into active metabolite through CYP2D6 is compromised [5]. To assure optimal pain control, tramadol therapy was discontinued and tapentadol was introduced, which doesn't have relevant drug-gene interactions [92]. Furthermore, the patient's intermediate to normal CYP2C19 activity can lead to an increased exposure to pantoprazole. We therefore suggested a dose reduction for pantoprazole or alternatively, a substitution with rabeprazole, a drug that has normal plasma concentrations despite the patient's CYP2C19 genotype [93].

\section{Clinical case III}

A 55-year-old male patient with history of untreated hypertension, hyperlipidemia, glucose intolerance and chronic obstructive pulmonary disease suffered ST-elevation myocardial infarction after physical exertion. Upon hospital admission, coronarography revealed total stenosis of right coronary artery, which was then successfully treated with percutaneous coronary intervention. Creatine kinase (CK), C-reactive protein, erythrocyte sedimentation rate (ESR), white blood cells were significantly increased. The patient was treated with anticoagulants, antiarrhythmics, acetylsalicylic acid, antihypertensives and simvastatin $(80 \mathrm{mg} /$ day $)$. Several weeks later, the patient developed weakness of truncal muscles, most prominent in upper-body and neck, leading to head-drop and myasthenia-like weakness. CK values increased beyond the initial values and the other laboratory values normalized. EMG of trapezius showed myopathic changes, nerve conduction velocity (NCV) was normal. Serum CK was 850 U/L, while myoglobin in urine was positive, as well as antibodies for 3-hydroxy-3-methylglutaryl-coenzyme A reductase (HMGCR).

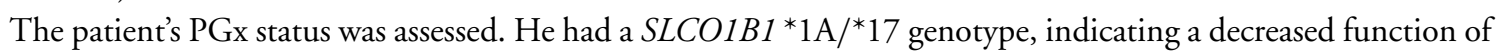
SLCO1B1 (intermediate metabolizer phenotype), with an increased risk of simvastatin-induced myopathy [32]. The findings suggested both muscle-toxicity with mitochondrial dysfunction and some sort of autoimmune mechanism involving HMGCR antibodies. Both have been described as side-effects of statin therapy (statins 'double-hit'). Simvastatin was, therefore, promptly removed from the therapy and L-carnitine and Co-Q10 introduced. 2 weeks later, the patient was able to hold up his head while walking (3-4/5) and his CK serum levels have normalized (S-CK $105 \mathrm{U} / \mathrm{L}$ ), but a certain level of weakness still remained, in oscillating severity.

\section{Clinical case IV}

A 49-year-old female patient was diagnosed with major depressive disorder in 1998, and since then has been on numerous antidepressants (ADs) without any clinical effect, including amitriptyline, maprotiline, venlafaxine, fluvoxamine, citalopram, sertraline, sulpiride and paroxetine which is the only AD she is taking now. In 2019, after 21 years of unsucessful treatment, she received PGx testing to determine if there were any significant drug-gene interactions (DGI).

PGx testing revealed a homozygous CYP2C19 genotype $2 /{ }^{*} 2$, associated with PM phenotype with no to very low enzyme activity, important for metabolizing amitriptyline, sertraline and citalopram [20,21]. Furthermore, venlafaxine showed moderate DGI. Due to polymorphisms in HRT2A and kainite GRIK4 encoding for serotonin and kainite receptors, there is significantly reduced likelihood of response to citalopram therapy [94]. CYP2D6 genotype $* 4 /{ }^{*} 35$, an important enzyme for metabolizing fluvoxamine and paroxetine, showed decreased activity [21]. These major and moderate DGIs, as well as receptor polymorphisms, could explain poor clinical outcomes. The patient was recommended to take bupropion and/or trazodone which do not undergo the aforementioned metabolic pathways and have minimal DGIs.

\section{Clinical case $\mathrm{V}$}

A 33-year-old chronic myeloid leukemia (CML) patient, came to the hospital for PGx testing. The patient was first diagnosed with CML in 2004 and had a history of ADRs to tyrosine kinase inhibitors (TKI) imatinib, nilotinib and dasatinib [95]. Seven years after the diagnosis of CML in 2004 and complete remission on imatinib, ADRs first occurred. The patient reported pain in the mandible and the extremities. The patient's therapy changed from imatinib to nilotinib, but generalized maculopapular rash presented. It was successfully treated with antihistamines. Nilotinib therapy was then discontinued due to planned pregnancy and the patient experienced a relapse of CML with BCR-ABL/ABL1 transcripts of 18.9\%. Dasatinib was introduced and CML was in remission. 2 years later, 
urine protein levels were elevated $(6.19 \mathrm{~g} / \mathrm{l})$, as well as erythrocyte sedimentation rate (ESR $=90 \mathrm{~mm} / 3.6 \mathrm{ks})$. The diagnosis of nephrotic syndrome was established. Consequently, a reduction to dasatinib dose brought urine protein level to normal. PGx testing determined that patient's CYP3A 4 enzyme genotype is wild type $\left({ }^{*} 1 /{ }^{*} 1\right)$ associated with extensive metabolism phenotype, which implies normal rates of TKI metabolism [96]. The finding was inconsistent with the observed nephrotic syndrome and other reported ADRs. A possible explanation would be a transient phenoconversion of CYP3A4 to PM phenotype, leading to accumulation of the drug in the patient's blood, which can be attributed to inflammation noted by elevated ESR, at the time of the nephrotic syndrome episode [97,98]. This case shows that indicating a broader approach that recognizes genetic, clinical and epigenomic factors is required to make an evidence-based decision on personalized therapy regimen, alongside further development of existing guidelines.

These several case reports show the importance of PGx in providing optimal treatment to patients. By doing PGx profiling in addition to analyzing other patient-related factors, we have not only prevented potentially severe ADRs but also assured the best possible therapy. PGx could change the whole paradigm of clinical specialists and contribute to modifications of their standard postulates by establishing every patient as an individual and thus, every treatment regimen as unique.

\section{The help of machine learning algorithm in providing best quality of care: experience from St Catherine Specialty Hospital}

As already discussed, the potential of applying AI in PGx is exciting, but challenging at the same time. Firstly, to create an efficient AI system, foundations are crucial. In the case of St Catherine Specialty Hospital, the foundations consist of two parts. First, the patient's general data and complete medical history including past and current medication use are obtained and transferred into an electronic database. The second part is the PGx report where genotype-predicted interactions for medications, as well as drug-drug interactions and other parameters are analyzed. The report also has to be stored electronically so it can be used at any given point in time. At St Catherine Specialty Hospital, these two components have been implemented in everyday clinical practice with interdisciplinary team consisting of physicians of various specialties and pharmacists. Furthermore, proactive (www.invitae.com/en/physician/category/CAT000043/) and diagnostic genetic health screening tests (www.invitae.com/en/physician/panelsgenes/), which analyze pathogenic genetic variants or variants of uncertain significance, related to cancers, cardiovascular diseases and some other disorders are also being applied in routine practice.

After patients leave the clinic, active tracking of their health status is very important. Tracking can be done by way of smartphone applications, smart watches or other medically approved devices, as well as by routine clinic visits. All changes in patient health and therapy status need to be updated, reported and stored in an electronic health record to complement the above mentioned patient-related PGx and medical report. Thus, over time, we will be able to gather data from thousands of patients in a single electronic record system, presenting an opportunity to apply AI principles. Along with active patient tracking and by programming AI to query these electronic data sets, we can train it to observe existing patterns, patient behaviors and health outcome changes and therefore make new patterns and find solutions to newly formed problems and clinical enigmas. This may include which drugs to use and and not use for a particular patient, at a particular time. We mustn't forget the research potential of AI. With proper training and data collection, AI can find novel correlations and patterns and give great momentum to researchers and future findings and discoveries.

\section{Conclusion \& future perspective}

Personalized medicine is the cornerstone of modern medicine. With so many hospital admissions being attributed to a 'one-size-fits-all' prescribing approach and ADRs being the fourth leading cause of death in the US [99], not to mention the huge economic implications this creates, a tailored approach for every patient is needed. At the center of this approach should be PGxs with the goal to improve drug safety and efficacy. Furthermore, therapy for each patient should be designed according to their personal characteristics, health status, lifestyle and pharmacogenetic profile.

There are still challenges, however. One of them is the implementation of PGx testing in clinical practice on a much broader level. If this were to be done, patients would easily present their PGx results to a healthcare institution and the goal of prescribing the right drug for the right patient at the right time would be more readily achieved. The help of AI specifically for PGx is needed so that large sums of data coming from patients who do PGx tests are 
analyzed altogether rather than case-by-case as it is done today. The results of the application of AI will promote a better understanding of the heterogeneity in processes that contribute to how a patient reacts to a specific drug at a specific time and dose, thus; allowing prescribers to tailor, or 'personalize', medicines to the nuanced and often unique features possessed by individual patients [100].

Not only would this make the process faster, but new data on genomic variations, drug-gene, drug-drug interactions and more could emerge, which could lead to new research and even better quality and more costeffective healthcare. Other challenges include the training of physicians who would need to interpret the PGx results with AI assistance. Furthermore, ethical questions, along with protected health information breaches emerge as a potential problem. Proper governmental legislation, policy and advocacy are needed to mitigate such risks. Additionally, the shortcomings of PGx which are seen with the phenomenon of phenoconversion and epigenetics still need to be addressed by the scientific community.

Although more effort is needed to implement PGx in today's clinical practice on a broader scale, there's undeniable evidence that personalized medicine is the treatment approach of the future and that PGx is at the center of it, with the benefits of cost reductions and improved quality of care which every healthcare system should value. Though the development of AI-based technologies in biomedicine is evolving rapidly, its implementation into every day patient care has not yet become a reality. Therefore, this is an evolutionary not a revolutionary process. We should embrace the challenges ahead of us and use the available tools to pioneer the application of AI principles to PGx-guided treatment. This is how future treatments will be made safer, more effective and cost-effective which, in turn, will benefit us all.

\section{Executive summary}

The role of pharmacogenomics in therapy optimization: opportunities \& limitations

- The ultimate aim of personalized medicine is to tailor medical treatment to the individual characteristics of each patient. Pharmacogenomic (PGx) testing has been identified as cost-effective in treating patients with drugs like irinotecan, azathioprine, warfarin, citalopram and others. Thus, PGx-guided treatment can be cost-effective and even a cost-saving strategy.

- Even though PGx does provide valuable insights, the impact of environmental factors noticeable in epigenetic changes and phenoconversion should also be included in the personalized medicine narrative.

Big Data in biomedicine: the emerging role of artificial intelligence

- An increasing amount of data relevant to disease symptoms, diagnostics, biomarkers, therapy, adverse effects - among others, has been collected largely due to the availability of various diagnostic and therapeutic tools and global informatization.

- In biomedicine artificial intelligence can provide benefits: in diagnostics; therapy management and risk predictions; for patients so they can monitor their health status; and for healthcare systems in providing money, time and personnel savings.

- Al requires the collection of very large data sets, legislative support and well-programmed algorithms in order to optimize all the tools currently available or in development, which are still lacking.

Overview of PGx testing in St Catherine Specialty Hospital

- St Catherine Specialty Hospital has embraced the personalized medicine concept striving to design the best available treatment to the patients based on their genotype.

- Patients receive detailed written and oral information about the testing protocol and are asked to sign an informed consent form.

- The RightMed ${ }^{\circledR}$ Comprehensive test panel covers 27 genes (CYP1A2, CYP2B6, CYP2C Cluster, CYP2C9, CYP2C19, CYP2D6, CYP3A4, CYP3A5, CYP4F2, COMT, DPYD, DRD2, F2, F5, GRIK4, HLA-A, HLA-B, HTR2A, HTR2C, IL28B (IFNL4), MTHFR, NUDT15, OPRM1, SLC6A4, SLCO1B1, TPMT, UGT1A1 and VKORC1) and provides genotype-derived guidance for more than 300 commonly prescribed medications. The obtained genetic data is interpreted by St Catherine's counseling team who additionally take into consideration the patient's personal, disease and therapy history to recommend the best quality of care. The counseling team makes use of the clinical decision support tool, RightMed Advisor, to look at potential drug-drug and drug-drug-gene interactions.

Conclusion \& future perspective

- Despite some limitations of artificial intelligence at the current time, contemporary medicine should remain open to the advancement of new technologies and try to implement them in a patient-centered approach while still retaining a responsible sense of objectivity.

- PGx Al concept should be integrated into Electronic Health Records to provide decision support as well as to reduce clinician burnout and improve patient care.

- Moving forward, future treatments should be safer, more effective and cost-effective which will, in turn, benefit all of society. 
Financial \& competing interests disclosure

St Catherine Specialty Hospital (represented by D Primorac, A Skelin, I Erceg Ivkošić, I Mikula, and D Erceg) is partnering with OneOme LLC (represented by Bernard Esquivel and Morgan Donaldson), offering the RightMed Comprehensive PGx test panel. K Žunić is an employee of PLIVA. The authors have no other relevant affiliations or financialinvolvement with any organization or entity with a financial interest in or financial conflict with the subject matter or materials discussed in the manuscript apart from those disclosed.

No writing assistance was utilized in the production of this manuscript.

Ethical conduct of research

The patients whose cases are presented in the text have signed Informed consent. The Principles outlined in the Declaration of Helsinki have been followed.

\section{References}

Papers of special note have been highlighted as: $\bullet$ of interest $\bullet \bullet$ of considerable interest

1. NHS England. Improving outcomes through personalized medicine 2016 (2016). www.england.nhs.uk/wp-content/uploads/2016/09/improving-outcomes-personalised-medicine.pdf

2. Agyeman AA, Ofori-Asenso R. Perspective: does personalized medicine hold the future for medicine? J. Pharm. Bioallied. Sci. 7(3), 239-244 (2015).

3. Novelli G. Personalized genomic medicine. Intern. Emerg. Med. 5(Suppl. 1), S81-S90 (2010).

4. Hood L, Friend SH. Predictive, personalized, preventive, participatory (P4) cancer medicine. Nat. Rev. Clin. Oncol. 8(3), 184-7 (2011).

- Describes the perspective of P4 medicine and challenges and technical and societal barriers in achieving this approach.

5. Bach-Rojecky L, Vađunec D, Žunić K et al. Continuing war on pain: a personalized approach to the therapy with nonsteroidal anti-inflammatory drugs and opioids. Per. Med. 16(2), 171-184 (2019).

6. Primorac D, Bach-Rojecky L. Could a personalized approach to therapy end the war on pain? Pract. Pain Manag. 19(3), 50-53 (2019).

7. Pizzo PA, Clark NM. Alleviating suffering 101 - pain relief in the United States. N. Engl. J. Med. 366(3), 197-199 (2012).

8. Lazarou J, Pomeranz BH, Corey PN. Incidence of adverse drug reactions in hospitalized patients: a meta-analysis of prospective studies. JAMA 279(15), 1200-1205 (1998).

-. This meta-analyses stresses that the incidence of serious and fatal adverse drug reactions in US hospitals is extremely high; it has been estimated that adverse drug reactions (ADRs) are between the fourth and sixth leading cause of death.

9. Plumpton CO, Roberts D, Pirmohamed M, Hughes DA. A systematic review of economic evaluations of pharmacogenetic testing for prevention of adverse drug reaction. Pharmacoeconomics 34(8), 771-793 (2016).

10. Spear BB, Heath-Chiozzi M, Huff J. Clinical application of pharmacogenetics. Trends Mol. Med. 7(5), 201-204 (2001).

11. Adams J. Pharmacogenomics and personalized medicine. Nat. Educ. 1(11), 194 (2008).

12. Kalow W, Tang BK, Endrenyi L. Hypothesis: comparisons of inter- and intra-individual variations can substitute for twin studies in drug research. Pharmacogenetics 8(4), 283-289 (1998).

13. Agyeman AA, Ofori-Asenso R. Perspective: does personalized medicine hold the future for medicine? J. Pharm. Bioallied Sci. 7(3), 239-244 (2015).

14. Osler W. On the educational value of the medical society. In: Aequanimitas with Other Addresses to Medical Students, Nurses and Practitioners of Medicine. P Blakiston's Son \& Co, PA, USA, 343-362 (1904).

15. Hong KW, Oh BM. Overview of personalized medicine in the disease genomic era. BMB Rep. 43(10), 643-648 (2010).

16. Tian Q, Price ND, Hood L. Systems cancer medicine: towards realization of predictive, personalized, preventive and participatory (P4) medicine. J. Intern. Med. 271(2), 111-121 (2012).

17. Thorn CF, Klein TE, Altman RB. PharmGKB: the pharmacogenomics knowledge base. Methods Mol. Biol. 1015, 311-320 (2013).

18. Caudle KE, Rettie AE, Whirl-Carrillo M et al. Clinical Pharmacogenetics Implementation Consortium guidelines for CYP2C9 and HLA-B genotypes and phenytoin dosing. Clin Pharmacol Ther. 96(5), 542-548 (2014).

19. Johnson JA, Caudle KE, Gong L et al. Clinical Pharmacogenetics Implementation Consortium (CPIC) guideline for pharmacogenetics-guided warfarin dosing: 2017 update. Clin. Pharmacol. Ther. 102(3), 397-404 (2017).

20. Hicks JK, Sangkuhl K, Swen JJ et al. Clinical Pharmacogenetics Implementation Consortium Guideline (CPIC) for CYP2D6 and CYP2C19 genotypes and dosing of tricyclic antidepressants: 2016 update. Clin. Pharmacol. Ther. 102(1), 37-44 (2017).

21. Hicks JK, Bishop JR, Sangkuhl K et al. Clinical Pharmacogenetics Implementation Consortium (CPIC) guideline for CYP2D6 and CYP2C19 genotypes and dosing of selective serotonin reuptake inhibitors. Clin. Pharmacol. Ther. 98(2), 127-134 (2015).

22. Scott SA, Sangkuhl K, Stein CM et al. Consortium guidelines for CYP2C19 genotype and clopidogrel therapy: 2013 update. Clin. Pharmacol. Ther. 94(3), 317-323 (2013). 
23. Moriyama B, Obeng AO, Barbarino J et al. Clinical Pharmacogenetics Implementation Consortium (CPIC) guidelines for CYP2C19 and voriconazole therapy. Clin. Pharmacol. Ther. 102(1), 45-51 (2017).

24. Brown JT, Bishop JR, Sangkuhl K et al. Clinical Pharmacogenetics Implementation Consortium (CPIC) guideline for CYP2D6 genotype and atomoxetine therapy. Clin. Pharmacol. Ther. 106(1), 94-102 (2019).

25. Crews KR, Gaedigk A, Dunnenberger HM et al. Clinical Pharmacogenetics Implementation Consortium guidelines for cytochrome P450 2D6 genotype and codeine therapy: 2014 update. Clin. Pharmacol. Ther. 95(4), 376-82 (2014).

26. Bell GC, Caudle KE, Whirl-Carrillo M et al. Clinical Pharmacogenetics Implementation Consortium (CPIC) guideline for CYP2D6 genotype and use of ondansetron and tropisetron. Clin. Pharmacol. Ther. 102(2), 213-218 (2017).

27. Goetz MP, Sangkuhl K, Guchelaar HJ et al. Clinical Pharmacogenetics Implementation Consortium (CPIC) guideline for CYP2D6 and tamoxifen therapy. Clin. Pharmacol. Ther. 103(5), 770-777 (2018).

28. Birdwell KA, Decker B, Barbarino JM et al. Clinical Pharmacogenetics Implementation Consortium (CPIC) guidelines for CYP3A5 genotype and tacrolimus dosing. Clin. Pharmacol. Ther. 98(1), 19-24 (2015).

29. Gammal RS, Court MH, Haidar CE et al. Clinical Pharmacogenetics Implementation Consortium (CPIC) guideline for UGT1A1 and atazanavir prescribing. Clin. Pharmacol. Ther. 99(4), 363-369 (2016).

30. Relling MV, Schwab M, Whirl-Carrillo M et al. Clinical Pharmacogenetics Implementation Consortium Guideline for thiopurine dosing based on TPMT and NUDT15 genotypes: 2018 update. Clin. Pharmacol. Ther. 105(5), 1095-1105 (2018).

31. Amstutz U, Henricks LM, Offer SM et al. Clinical Pharmacogenetics Implementation Consortium (CPIC) guideline for dihydropyrimidine dehydrogenase genotype and fluoropyrimidine dosing: 2017 update. Clin. Pharmacol. Ther. 103(2), $210-216$ (2017).

32. Ramsey LB, Johnson SG, Caudle KE et al. The Clinical Pharmacogenetics Implementation Consortium Guideline for SLCO1B1 and simvastatin-induced myopathy: 2014 update. Clin. Pharmacol. Ther. 96(4), 423-428 (2014).

33. Clancy JP, Johnson SG, Yee SW et al. Clinical Pharmacogenetics Implementation Consortium (CPIC) guidelines for ivacaftor therapy in the context of CFTR genotype. Clin. Pharmacol. Ther. 95(6), 592-597 (2014).

34. Phillips EJ, Sukasem C, Whirl-Carrillo M et al. Clinical Pharmacogenetics Implementation Consortium guideline for HLA genotype and use of carbamazepine and oxcarbazepine: 2017 update. Clin. Pharmacol. Ther. 103(4), 574-581 (2018).

35. Martin MA, Klein TE, Dong BJ, Pirmohamed M, Haas DW, Kroetz DL. Clinical Pharmacogenetics Implementation Consortium Guidelines for HLA-B genotype and abacavir dosing. Clin. Pharmacol. Ther. 91(4), 734-738 (2012).

36. Hershfield MS, Callaghan JT, Tassaneeyakul W et al. Clinical Pharmacogenetics Implementation Consortium guidelines for human leukocyte antigen-B genotype and allopurinol dosing. Clin. Pharmacol. Ther. 93(2), 153-158 (2013).

37. Muir AJ, Gong L, Johnson SG et al. Clinical Pharmacogenetics Implementation Consortium (CPIC) guidelines for IFNL3 (IL28B) genotype and PEG interferon- $\alpha$-based regimens. Clin. Pharmacol. Ther. 95(2), 141-146 (2014).

38. Relling MV, McDonagh EM, Chang T et al. Clinical Pharmacogenetics Implementation Consortium. Clinical Pharmacogenetics Implementation Consortium (CPIC) guidelines for rasburicase therapy in the context of G6PD deficiency genotype. Clin. Pharmacol. Ther. 96(2), 169-174 (2014).

39. Gonsalves SG, Dirksen RT, Sanghul K et al. Clinical Pharmacogenetics Implementation Consortium (CPIC) guideline for the use of potent volatile anesthetic agents and succinylcholine in the context of RYR1 and CACNA1S genotypes. Clin. Pharmacol. Ther. 105(6), 1338-1344 (2019).

40. Clinical Pharmacogenetics Implementation Consortium 2019 (2019). https://cpicpgx.org/

41. Aneesh TP, Sonal Sekhar M, Jose A, Chandran L, Zachariah SM. Pharmacogenomics: the right drug to the right person. J. Clin. Med. Res. 1(4), 191-194 (2009).

42. Issa NT, Wathieu H, Ojo A, Byers SW, Dakshanamurthy S. Drug metabolism in preclinical drug development: a survey of the discovery process, toxicology, and computational tools. Curr. Drug Metab. 18(6), 556-565 (2017).

43. Chawla NV, Davis DA. Bringing big data to personalized healthcare: a patient-centered framework. J. Gen. Intern. Med. 28(Suppl 3), S660-S665 (2013).

44. Gartner Glossary, Big data 2019 (2019). www.gartner.com/en/information-technology/glossary/big-data

45. Topol EJ. High-performance medicine: the convergence of human and artificial intelligence. Nat. Med. 25(1), 44-56 (2019).

- Discusses benefits of artificial intelligence (AI) for clinicians, patients and health system as well as current limitations, including bias, privacy and security, and lack of transparency, along with the future directions of AI-based technologies applications.

46. He J, Baxter SL, Xu J, Xu J, Zhou X, Zhang K. The practical implementation of artificial intelligence technologies in medicine. Nat. Med. 25(1), 30-36 (2019).

47. Turing AM. Computing machinery and intelligence. Mind 59, 433-460 (1950).

48. Servan-Schreiber D. Artificial intelligence and psychiatry. J. Nerv. Ment. Dis. 74(4), 191-202 (1986).

49. Hassabis D. Artificial intelligence: chess match of the century. Nature 544, 413-414 (2017).

50. Mohammed M, Khan M, Bashier E. Machine learning: algorithms and applications. 10.1201/9781315371658 (2016). 
51. Hessler G, Baringhaus KH. Artificial intelligence in drug design. Molecules 23(10) (2018).

52. Hosny A, Parmar C, Quackenbush J, Schwartz LH, Aerts HJWL. Artificial intelligence in radiology. Nat. Rev. Cancer 18(8), 500-510 (2018).

53. Esteva A, Robicquet A, Ramsundar B et al. A guide to deep learning in healthcare. Nat Med. 25(1), 24-29 (2019).

- The article presents deep-learning techniques for healthcare, focusing on deep learning in computer vision for medical imaging, natural language processing, reinforcement learning in the context of robotic-assisted surgery, and generalized methods for genomics.

54. Kalinin AA, Higgins GA, Reamaroon N et al. Deep learning in pharmacogenomics: from gene regulation to patient stratification. Pharmacogenomics 19(7), 629-650 (2018).

55. Labovitz DL, Shafner L, Reyes Gil M, Virmani D, Hanina A. Using artificial intelligence to reduce the risk of nonadherence in patients on anticoagulation therapy. Stroke 48(5), 1416-1419 (2017).

56. Bughin J, Hazan E, Ramaswamy S et al. Artificial intelligence the next digital frontier? McKinsey and Company Global Institute (2017). www.mckinsey.com/ /media/McKinsey/Industries/AdvancedElectronics/OurInsights/Howartificialintelligencecandeliverrealvaluetoco mpanies/MGI-Artificial-Intelligence-Discussion-paper.ashx.

57. Raja K, Patrick M, Elder JT, Tsoi LC. Machine learning workflow to enhance predictions of adverse drug reactions (ADRs) through drug-gene interactions: application to drugs for cutaneous diseases. Sci Rep. 7, 3690 (2017).

58. Seyhan AA, Carini C. Are innovation and new technologies in precision medicine paving a new era in patients centric care? J. Transl. Med. 17(1), 114 (2019).

59. Wod M. Trial by artificial intelligence. Nature. 573(7775), S100-S102 (2019).

60. Shah P, Kendall F, Khozin S et al. Artificial intelligence and machine learning in clinical development: a translational perspective. NPJ Digit Med. 2, 69 (2019).

61. Pesapane F, Codari M, Sardanelli F. Artificial intelligence in medical imaging: threat or opportunity? Radiologists again at the forefront of innovation in medicine. Eur. Radiol. Exp. 2(1), 35 (2018).

62. Athreya AP, Neavin D, Carrillo-Roa T et al. Pharmacogenomics-driven prediction of antidepressant treatment outcomes: a machine-learning approach with multi-trial replication. Clin. Pharmacol. Ther. doi: 10.1002/cpt.1482 (2019) (Epub ahead of print).

63. Tai AMY, Albuquerque A, Carmona NE et al. Machine learning and big data: Implications for disease modeling and therapeutic discovery in psychiatry. Artif. Intell. Med. 99 (2019).

64. Kim YJ, Kelley BP, Nasser JS, Chung KC. Implementing precision medicine and artificial intelligence in plastic surgery: concepts and future prospects. Plast. Reconstr. Surg. Glob. Open. 7(3), e2113 (2019).

65. Thomford NE, Bope CD, Agamah FE et al. Implementing artificial intelligence and digital health in resource-limited settings? Top 10 lessons we learned in congenital heart defects and cardiology. OMICS. doi: 10.1089/omi.2019.0142 (2019) (Epub ahead of print).

66. IBM's Watson supercomputer recommended 'unsafe and incorrect' cancer treatments, internal documents show (2018). www.statnews.com/2018/07/25/ibm-watson-recommended-unsafe-incorrect-treatments/.

67. Nadarzynski T, Miles O, Cowie A, Ridge D. Acceptability of artificial intelligence (AI)-led chatbot services in healthcare: a mixed-methods study. Digit Health. 5, 2055207619871808 (2019).

68. Sarwar S, Dent A, Faust K et al. Physician perspectives on integration of artificial intelligence into diagnostic pathology. NPJ Digit. Med. 2, 28 (2019).

69. Ašić A, Marjanović D, Mirat J, Primorac D. Pharmacogenetics of novel oral anticoagulants: a review of identified gene variants $\&$ future perspectives. Per. Med. 15(3), 209-221 (2018).

70. Cosgun E, Limdi NA, Duarte CW. High-dimensional pharmacogenetic prediction of a continuous trait using machine learning techniques with application to warfarin dose prediction in African Americans. Bioinformatics 27(10), 1384-1389 (2011).

71. Su SC, Hung SI, Fan WL, Dao RL, Chung WH. Severe cutaneous adverse reactions: the pharmacogenomics from research to clinical implementation. Int J Mol Sci. 17(11), 1890 (2016).

72. Velez-Velez LM, Hughes CL, Kasi PM. Clinical value of pharmacogenomic testing in a patient receiving FOLFIRINOX for pancreatic adenocarcinoma. Front Pharmacol. 9, 1309 (2018).

73. Chan SL, Samaranayake N, Ross CJ et al. Genetic diversity of variants involved in drug response and metabolism in Sri Lankan populations: implications for clinical implementation of pharmacogenomics. Pharmacogenet. Genomics 26(1), 28-39 (2016).

74. Ramos E, Doumatey A, Elkahloun AG et al. Pharmacogenomics, ancestry and clinical decision making for global populations. Pharmacogenomics J. 14(3), 217-222 (2014).

75. Ahn E, Park T. Analysis of population-specific pharmacogenomic variants using next-generation sequencing data. Sci Rep. 7, 8416 (2017).

76. Cavalli G, Heard E. Advances in epigenetics link genetics to the environment and disease. Nature. 571, 489-499 (2019).

77. Shah RR, Smith RL. Addressing phenoconversion: the Achilles' heel of personalized medicine. Br J Clin Pharmacol. 79(2), 222-240 (2015). 
- This review focuses on drug-induced phenoconversion and discusses potential approaches to rectify some of the current shortcomings. It identifies principal challenges facing personalized medicine, which include identification of the drugs that are susceptible to phenoconversion; comedications that can cause phenoconversion; and dosage amendments that need to be applied during and following phenoconversion.

78. Sultana J, Cutroneo P, Trifirò G. Clinical and economic burden of adverse drug reactions. J. Pharmacol. Pharmacother. 4(Suppl. 1), S73-S77 (2013).

79. Berm EJJ, Looff MD, Wilffert B, Boersma C, Annemans L, Vegter S et al. Economic evaluations of pharmacogenetic and pharmacogenomic screening tests: a systematic review. second update of the literature. PLoS ONE 11(1), e0146262 (2016).

80. Maciel A, Cullors A, Alukowiak A, Garces J. Estimating cost savings of pharmacogenetic testing for depression in real-world clinical settings. Neuropsychiatr Dis Treat. 14(1), 225-230 (2018).

81. World Health Organization. Depression Fact Sheet. www.who.int/

82. Mrazek DA, Hornberger JC, Altar CA, Degtiar I. A review of the clinical, economic, and societal burden of treatment-resistant depression: 1996-2013. Psychiatr Serv. 65(8), 977-987 (2014).

83. Martinson M, Koep T. The OneOme RightMed comprehensive test: a Markov cost analysis of optimizing pharmaceutical therapy. A White Paper developed by Technomics Research \& OneOme LLC (2018).

84. Gold HT, Hall MJ, Blinder V, Schackman BR. Cost effectiveness of pharmacogenetic testing for uridine diphosphate glucuronosyltransferase 1A1 before irinotecan administration for metastatic colorectal cancer. Cancer. 115(17), 3858-3867 (2009).

85. Verbelen M, Weale ME, Lewis CM. Cost-effectiveness of pharmacogenetic-guided treatment: are we there yet? Pharmacogenomics J. 17(5), 395-402 (2017).

- The authors review economic evaluations for PGx by estimating the proportion of evaluations that found PGx-guided treatment to be cost-effective or dominant over the alternative strategies. This analysis found PGx-guided treatment as a cost-effective and even a cost-saving strategy.

86. Bach-Rojecky L, Vađunec D, Lozić M et al. Challenges in anesthesia personalization - resolving the pharmacogenomics puzzle. Per. Med. doi:10.2217/pme-2019-0056 (2019) (Epub ahead of print).

87. Fröhlich H, Balling R, Beerenwinkel $\mathrm{N}$ et al. From hype to reality: data science enabling personalized medicine. BMC Med. 16(1), 150 (2018).

88. OneOme. www.oneome.com

89. O’Donoghue ML, Braunwald E, Antman EM et al. Pharmacodynamic effect and clinical efficacy of clopidogrel and prasugrel with or without a proton-pump inhibitor: an analysis of two randomised trials. Lancet 374(9694), 989-997 (2009).

90. Pinheiro LF, Franca CN, Izar MC et al. Pharmacokinetic interactions between clopidogrel and rosuvastatin: effects on vascular protection in subjects with coronary heart disease. Int. J. Cardiol. 158(1), 125-129 (2012).

91. Herings RM, de Boer A, Stricker BH, Leufkens HG, Porsius A. Hypoglycaemia associated with use of inhibitors of angiotensin converting enzyme. Lancet 345(8959), 1195-1198 (1995).

92. Barbosa J, Faria J, Queiros O, Moreira R, Carvalho F, Dinis-Oliveiira RJ. Comparative metabolism of tramadol and tapentadol: a toxicological perspective. Drug Metab. Rev. 48(4), 577-592 (2016).

93. El Rouby N, Lima JJ, Johnson JA. Proton pump inhibitors: from CYP2C19 pharmacogenetics to precision medicine. Expert Opin. Drug Metab. Toxicol. 14(4), 447-460 (2018).

94. Tansey KE, Guipponi M, Hu X et al. Contribution of common genetic variants to antidepressant response. Biol. Psychiatry 73(7), 679-682 (2013).

95. Mandac Rogulj I, Matišić V, Arsov B et al. Dasatinib-induced nephrotic syndrome: a case of phenoconversion. CMJ 60, 250-254 (2019).

96. Teo YL, Ho HK, Chan A. Metabolism-related pharmacokinetic drug-drug interactions with tyrosine kinase inhibitors: current understanding, challenges and recommendations. Br. J. Clin. Pharmacol. 79(2), 241-253 (2015).

97. Shah RR, Smith RL. Inflammation-induced phenoconversion of polymorphic drug metabolizing enzymes: hypothesis with implications for personalized medicine. Drug Metab. Dispos. 43(3), 400-410 (2015).

98. Shah RR. Pharmacogenetics and precision medicine: is inflammation a covert threat to effective genotype-based therapy? Ther. Adv. Drug Saf. 8(9), 267-272 (2017).

99. Preventable Adverse Drug Reactions: A Focus on Drug Interactions 2018 (2018). www.fda.gov/drugs/drug-interactions-labeling/preventable-adverse-drug-reactions-focus-drug-interactions

100. Schork NJ. Artificial intelligence and personalized medicine. In: Von Hoff D, Han H (Eds). Precision Medicine in Cancer Therapy. Cancer Treatment and Research. Cham, Switzerland, Springer (2019). 